\title{
Infant with SVT: Case Report
}

\author{
BIPLOB BHATTACHARJEE ${ }^{1}$, RAJAT SANKER ROY BISWAS ${ }^{1}$, MD. SALAH UDDIN ${ }^{2}$, MD. MUKHLESUR RAHMAN ${ }^{3}$, \\ MD. KHURSHED AHMED ${ }^{3}$, MD. ASHRAF UDDIN SULTAN ${ }^{3}$ \\ ${ }^{1}$ Department of Cardiology, Chittagong Medical College, Chittagong, ${ }^{2}$ Department of Pharmacology, Bangladesh Medical \\ College, Dhaka ${ }^{3}$ Department of Cardiology, Bangabandhu Sheikh Mujib Medical University, Dhaka
}

Address of Correspondence : Dr. Biplob Bhattacharjee, Assistant Professor of Cardiology, Chittagong Medical College, E-mail:

\begin{abstract}
:
A one and half year old child presented with lethargy and unwilling to take food for 2 days. He had history of unusual cry with a history of pneumonia 2 month back. After getting high heart rate on auscultation, a 12 - lead ECG was done and diagnosed as a case of supra ventricular tachycardia (SVT). He was managed by using injection adenosine intravenously by which he was reverted to sinus rhythm. In this case, the treatment strategies of SVT in children will be discussed with other option also.
\end{abstract}

\section{Introduction:}

SVT or paroxysmal atrial tachycardia or paroxysmal SVT is the most common abnormal tachycardia in children with an incidence of 1:250 to $1: 1000 .{ }^{1}$ In $90 \%$ cases it is due to reentrant tachycardia in otherwise a normal healthy child. ${ }^{2}$ Here the rate is usually more than 220 beats per minute and affecting both upper and lower chamber of the heart. ${ }^{3}$ The child is usually seen fussy and sleepy than normal. Sometimes SVT can be detected while the baby is still in the mothers womb. ${ }^{4}$ In that case, mother will take the pin for her baby. SVT is not a life threatening condition. Treatment is only considered if episodes are prolonged or frequent. For many infants SVT is a time limited problem. So treatment with medication could otherwise be stopped after six to 12 months. ${ }^{5}$

\section{Case note:}

A baby of one and half years from Cox's Bazar, admitted at Chittagong Medical College Hospital cardiology department through emergency on 28,02,10 with the complaints of frequent cry, lethargy, and unwilling to take food for two days. According to his mothers statement her baby was alright three months back. Then he developed respiratory tract infection which was managed by local doctor with bronchodilator and steroid with little improvement. Later he was given antibiotics for two weeks and his symptoms relieved. Then after one month he again developed restlessness, occasional excessive cry with increased frequency and duration. His mother noticed feeling of excessive heart beat while he was in her womb. She noticed his heart beat was very rapid and at time he became lethargic and refused to take food. She consulted with a pediatrician about the problem and the consultant diagnosed it as a case of suraventricular tachycardia after an ECG and referred the patient to the $\mathrm{CMCH}$. The birth of the baby was uneventful and he was duly immunized and he came from a low socioeconomic status family.

At $\mathrm{CMCH}$ the patient was examined thoroughly. He was found ill looking lethargic, with $10 \mathrm{~kg}$ weight and BCG mark on left arm. He had no anaemia, jaundice or cyanosis, his heart rate was $214 \mathrm{bpm}$, regular, respiratory rate was 44breaths/min and heart sounds were difficult to differentiate due to high rate. He had no audible murmur and lung base was clear on auscultation. In full blood count he had only neutrophilic leucocytosis with ESR- $40 \mathrm{~mm}$ in $1^{\text {st }}$ hour. Chest $\mathrm{X}$-ray showed mild cardiomegaly and a 12 lead ECG showed a run of SVT (Figure-1) and dilatation of RA, RV with mild TR found in echocardiography.

Patient was treated according to the protocol of haemodynamically stable SVT. The patient was attached with cardiac monitor and $2.5 \mathrm{mg}$ of diazepam was given per rectally and carotid sinus massage was tried simultaneously. As rhythm was not reverted to normal gargling and gagging with induced vomiting was also tried to revert it. But as all failed Inj Adenosine $0.5 \mathrm{mg}(0.05 \mathrm{mg} / \mathrm{kg}$ ) was given intravenously as bolus first with no change. Again a bolus of $1 \mathrm{mg}(.2 \mathrm{mg} / \mathrm{kg})$ was given intravenously which reverted the rhythm in to normal seen in the cardiac monitor. It also showed the rate reduced to $138 \mathrm{bpm}$. On auscultation both heart sound became audible with clear lung base.

Mother noticed no more excessive heart beat and the baby became cheerful after some time.

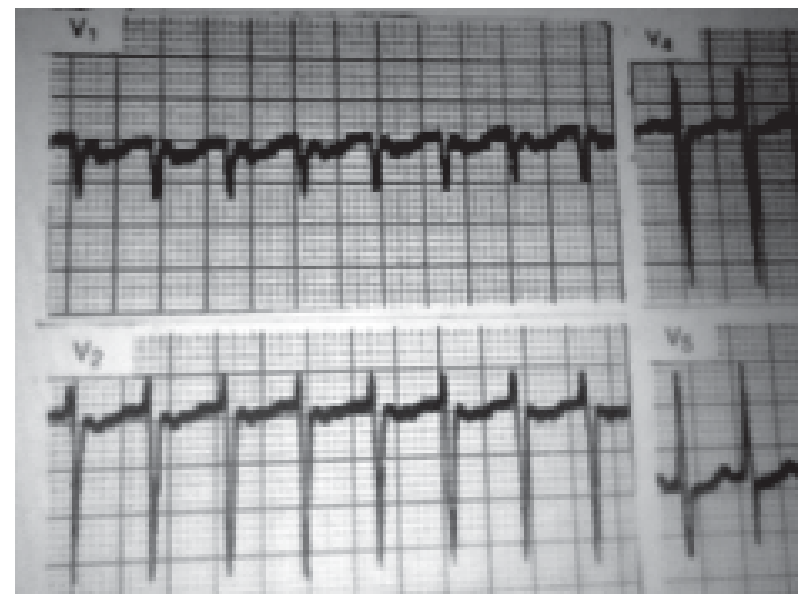

Fig.-1: ECG shows SVT. 


\section{Discussion:}

Supraventricular tachycardia ia a variety of rhythm disturbances that arise in the atria and $\mathrm{AV}$ juncional area (AV node and adjacent specialized tissue) which result from either increased automaticity or re-entry.

Increased automaticity includes-

1. Atrial tachycardia

2. Jnctional or nodal tachycardia

3. Multifocal or chaotic atrial tachycardia

Re-entry include-

1. Arial flutter

2. Atrial fibrillation

3. AV nodal re-entry tachyacardia (AVNRT)

4. AV nodal re-entry tachyacardia with WPW syndrome

So, in the broadest sense, the term parosxismal SVT refers to any recurrent SVT where arrhythmia originating within atrium and characterized by-

1. Starting abruptly

2. Narrow QRS complex- closely resembling sinus rhythm

3. Rate 140-220bpm

4. But broadening of complex may be present in SVT with bundle branch block and SVT with aberrant conduction,

Diagnosis:

1. Palpitation, chest pain, dyspnoea, lethargy

2. Sign/ symptoms of heart failure may or may not be present

3. There may be structural heart disease, like

- Ebstein anomaly

- Arrythmogenic right ventricular dysplasia

- Transposition of great vessel

Investigation:

1. 12 lead ECG gold standard

2. Holter monitoring

3. Event recorder of 30 days.

4. Electrophysiological study of aberrant pathways

5. To find out the causes the following investigations should be considered

- $\quad$ Serum TSH

- Serum electrolytes

- CXR PAview

- Cardiac echo
Differential diagnosis of SVT:

If QRS complex are narrow-

1. Atrial tachycardia

2. Atrial fibrillation

3. Arial flutter

If QRS complex are wide-

1. SVT with functional bundle branch block

2. SVT with aberrant conduction

3. Ventricular tachycardia

Treatment of SVT:

Acute case in haemodynamically stable patient the following can be done:

1. Non- pharmacological measures-

- Valsalva maneuvers

- Ice cool compression over parotid gland

- Carotid sinus massage

2. Pharmacological measures:

- A. Inj Adenosine, a purinergic blocking agent causes acute and transient blockade of AV node, is the drug of choice for acute termination of SVT. Several studies indicate that adenosine is about 100 percent effective. It has rapid onset of action (within seconds) and a short half life (10 sec) and so given with 15-10 ml saline flush and it does not impair contractility.

- According to Americal Heart Association the dosage of Adenosine is $0.1-0.3 \mathrm{mg} / \mathrm{kg} /$ dose with gradual increment of $0.05 \mathrm{mg} / \mathrm{kg}$ after 2 sec interval

- Minor side effects include- dyspnoea and chest pain. Adenosine may cause sinus arrest or bradycardia but resolves quickly. After termination of PSVT atrial and ventricular premature beat may be seen. But in patients with long QT syndrome a fatal polymorphic ventricular arrhythmia may be seen. It shortens the atrial refractory period and may precipitate atrial fibrillation. In this case repeat administration of adenosine or calcium channel blocker may be effective.

- B. Verapamil: It is a calcium channel blocker which slows conduction both in the slow and fast pathways.

- Dosage: $0.4-0.8 \mathrm{mg} / \mathrm{kg} / \mathrm{day}$ in divided dose. A single bolus of Verapamil may be followed by additional one or two mg boluses 10 min apart if initial dose does not terminate the SVT. It is about $90 \%$ effective. It is contraindicated below one 
year of age and can cause ventricular asystole and precipitate heart failure.

- Other drugs that decrease vagal tone such as Digoxin, decrease sympathetic tone like âblockers and procainamide, propafenone, flecanide, sotalol and amiodarone also may be considered.

If patient is haemodynamically unstable -

1. Direct current cardioversion should be given-

- For cardioversion- $0.5-1 \mathrm{j} / \mathrm{kg}$

- For defrillation- $0.5-2 \mathrm{j} / \mathrm{kg}$

For long term management - Radiofrequency ablation is the gold standard strategy.

\section{Conclusion:}

AVNRT is not a life threatening arrhythmia, the primary indication for its treatment relates to its impact on patients quality of life. Patients, who develop a highly symptomatic episode of PSVT, particularly if it requires hospital admission, may elect to initiate therapy after single episode. In contrast, an asymptomatic patient with episode of PSVT that terminate spontaneously or with a valsalva maneuver may elect to be followed clinically without specific therapy.

\section{Refferences:}

1. Esdaile J M, Abrahamowicz15 Jan 2009 Types of arrhythmia in children- Available from www.americanheart.org/presenter

2. Bastian HM. Roseman JM et al Feb 2003- Tachyarrhythmias in infants and children- Available from www.starship.org.nz/ clinicalcivideline/starship children's health guideline

3. Popescue, Eonescu R posted by Cydia on May 05.1999 SVT in children. Available from www.medhelp.org/posts/heart

4. Robert I. et al, clinical assessment and management of arrhythmia and conduction disturbances- supraventricular arrhythmias. Hurts the heart. Mackgraw Hill company. 2001; 10(1): 804-5.

5. Francles M. et al, Harrison's Principles of Internal Medicine. Mackgraw Hill Company, 2008; 17: 1425-43. 